\title{
Racial Differences by Ischemic Stroke Subtype: A Comprehensive Diagnostic Approach
}

\author{
Sarah Song, ${ }^{1,2}$ Richard E. Burgess, ${ }^{3}$ and Chelsea S. Kidwell ${ }^{3}$ \\ ${ }^{1}$ Stroke Center and Department of Neurology, UCLA Medical Center, Los Angeles, CA 90095-6901, USA \\ ${ }^{2}$ American Heart Association/Pharmaceutical Roundtable-Spina Outcomes Research, Center at UCLA, Los Angeles, CA 90095, USA \\ ${ }^{3}$ Cerebrovascular Center, Cleveland Clinic Foundation, 9500 Euclid Avenue, Cleveland, OH 44195, USA
}

Correspondence should be addressed to Sarah Song, sarahsong@gmail.com

Received 2 November 2011; Revised 30 January 2012; Accepted 15 February 2012

Academic Editor: Daniel Woo

Copyright (c) 2012 Sarah Song et al. This is an open access article distributed under the Creative Commons Attribution License, which permits unrestricted use, distribution, and reproduction in any medium, provided the original work is properly cited.

\begin{abstract}
Background. Previous studies have suggested that black populations have more small-vessel and fewer cardioembolic strokes. We sought to analyze racial differences in ischemic stroke subtype employing a comprehensive diagnostic workup with magnetic resonance-imaging-(MRI-) based evaluation including diffusion-weighted imaging (DWI). Methods. 350 acute ischemic stroke patients admitted to an urban hospital with standardized comprehensive diagnostic evaluations were retrospectively analyzed. Ischemic stroke subtype was determined by three Trial of Org 10172 in Acute Stroke Treatment (TOAST) classification systems. Results. We found similar proportions of cardioembolic and lacunar strokes in the black and white cohort. The only subtype category with a significant difference by race was "stroke of other etiology," more common in whites. Black stroke patients were more likely to have an incomplete evaluation, but this did not reach significance. Conclusions. We found similar proportions by race of cardioembolic and lacunar strokes when employing a full diagnostic evaluation including DWI MRI. The relatively high rate of cardioembolism may have been underappreciated in black stroke patients when employing a CT approach to stroke subtype diagnosis. Further research is required to better understand the racial differences in frequency of "stroke of other etiology" and explore disparities in the extent of diagnostic evaluations.
\end{abstract}

\section{Introduction}

Prior studies have demonstrated a significantly higher incidence of both ischemic and hemorrhagic stroke in black patients compared to whites [1-3]. For ischemic stroke, population-based studies in North America have specifically reported an increased incidence of small vessel, large artery (intracranial), and undetermined stroke subtypes in the black population $[1,2,4,5]$. In addition, several studies have examined differences in the proportion or distribution of ischemic stroke subtypes by race. Specifically, these studies have found a greater proportion of small vessel strokes [6-8] and a counterbalancing smaller proportion of cardioembolic strokes $[1,2]$ in blacks as compared to whites.

These racial differences have been attributed, in part, to a higher prevalence of stroke risk factors in blacks, including smoking, hypertension, and diabetes [2]. However, there is the possibility that the incidence rates of certain subtypes of strokes, such as extracranial large artery, lacunar or cardioembolic subtypes, have been under- or overestimated in some studies due to incomplete diagnostic workup. In these previous studies, the majority of patients received computed tomography (CT) rather than MR imaging. This is important as other studies have shown that diffusion MRI can substantially improve the accuracy of the diagnosis of early ischemic stroke subtype $[9,10]$. In particular, small vessel and brainstem strokes may be missed on CT, and small, punctate lesions in multiple vascular territories (indicative of a proximal embolic source) may only be visualized on DWI. In this hospital-based study, we sought to examine the racial differences in the proportion of ischemic stroke subtypes as determined after a comprehensive diagnostic workup, including DWI MRI and vascular imaging.

\section{Methods}

Consecutive patients with a diagnosis of acute ischemic stroke admitted to or evaluated by the stroke service between 
TABLE 1: Baseline characteristics of the cohort as a whole and by race.

\begin{tabular}{|c|c|c|c|c|}
\hline & Overall $(n=350)$ & Black $(n=261)$ & White $(n=89)$ & $P$ value \\
\hline Age (mean) & 63 & 63 & 63 & 0.94 \\
\hline Male gender, $n(\%)$ & $171(49)$ & $109(42)$ & $62(70)$ & $<0.01$ \\
\hline Hypertension, $n(\%)$ & $272(78)$ & $213(82)$ & $59(66)$ & $<0.01$ \\
\hline Diabetes mellitus, $n(\%)$ & $133(38)$ & $108(41)$ & $25(28)$ & 0.03 \\
\hline Coronary artery disease, $n(\%)$ & $87(25)$ & $60(23)$ & $27(30)$ & 0.17 \\
\hline Hyperlipidemia, $n(\%)$ & $131(37)$ & $94(36)$ & $37(42)$ & 0.35 \\
\hline Tobacco use (current), $n(\%)$ & $64(17)$ & $43(16)$ & $18(20)$ & 0.42 \\
\hline
\end{tabular}

October 2004 and July 2007 were identified. The Washington Hospital Center is a large hospital in an urban setting serving the greater Washington D.C. area in which all patients presenting within 24 hours are evaluated emergently by the stroke service and those beyond 24 hours are typically seen as consults. For the current analysis, only patients with race identified as white (or Caucasian or non-Hispanic white), or black (or African American) were included. Patients with classically defined transient ischemic attacks (symptom resolution within 24 hours of onset) were excluded. As Washington Hospital Center is a large cardiology referral center in the DC metropolitan area, we excluded 25 patients with inhouse strokes to minimize bias from acute primary cardiac referrals.

As part of the standard stroke diagnostic workup, every patient received noninvasive vessel imaging of the head and neck (MRA or ultrasound); echocardiography (transthoracic, and if deemed necessary, transesophageal); a 12-lead electrocardiogram; routine laboratory studies, including fasting lipid panel and hemoglobin A1C. Patients were monitored in the inpatient telemetry unit throughout their admission to detect potential arrhythmias. If the patient was under 55 years old and no other source of stroke was found, a hypercoagulable panel was also sent. All patients received brain imaging via diffusion-weighted MRI (95\% of patients) or CT scan.

Presence of comorbid risk factors including hypertension, diabetes, tobacco usage, coronary artery disease, and hyperlipidemia was recorded and analyzed. These factors were either defined on admission by medical history documentation, patient report, documented medication for pertinent condition, or based on new diagnosis during in-hospital diagnostic evaluation. Race and ethnicity were classified using US Census categories. Ischemic strokes were categorized employing three approaches: the standard Trial of Org 10172 in Acute Stroke Treatment (TOAST) classification [11], the Stop Stroke Study (SSS) TOAST classification [12], and MR imaging modified TOAST [9]. The standard TOAST classification has five categories: large artery disease, small vessel disease, cardioembolic source, other, or unknown. The SSS TOAST classification uses the same basic five categories, but divides each group into evident, probable, or possible classifications, to help allow for multiple potential etiologies for ischemic stroke. Using a decision algorithm, a final determination of possible, probable, or evident etiology is made. The MR imaging modified TOAST classification utilizes MRA and DWI findings, along with clinical history and exam, to categorize patients into smallvessel disease stroke, cardioembolic stroke, large vessel atherothromboembolic stroke, other etiology (such as hypercoagulable state), unknown etiology, more than one likely etiology, and unknown stroke subtypes. One neurologist (SS) reviewed the chart, the imaging, and the diagnostic reports, to make determinations as to the TOAST subtypes. In equivocal cases, a second neurologist (C. S. Kidwell) assessed the information and a consensus was reached.

Differences in dichotomous variables were analyzed using chi-square analysis or Fisher's exact test. The student's $t$-test was used to analyze differences in the mean of continuous variables between groups. All statistical analyses were performed using the Statistical Package for the Social Sciences version 17.0 (SPSS, Chicago, IL, USA).

\section{Results}

A total of 350 patients meeting inclusion criteria were consecutively admitted with diagnoses of ischemic stroke to the academic acute stroke service at an innercity community hospital. Of these, 261 subjects were black and 89 subjects were white. Table 1 shows the baseline characteristics for the cohort as a whole and by race. Overall, mean age was 63 and $49 \%$ of subjects were male. Overall, $78 \%$ of subjects had a history of hypertension, 38\% diabetes mellitus, 25\% coronary artery disease, 37\% hyperlipidemia, and 17\% were current tobacco smokers. The black cohort was $42 \%$ male, compared to $70 \%$ in the white cohort $(P<0.01)$ and also had a greater frequency of hypertension $(82 \%$ versus $66 \%, P<$ $0.01)$ and diabetes mellitus (41\% versus $28 \%, P=0.03)$. Other risk factors were similar between the white and the black cohorts.

Table 2 and Figure 1 show the proportion of strokes by subtype and race for each of the three TOAST rating systems. For the standard TOAST, there was no significant difference by race for the proportion of strokes that were small vessel, cardioembolic, large artery, or undetermined. Stroke of other etiology was significantly more frequent in the white versus black group using all 3 classification systems (for standard TOAST $15 \%$ versus $7 \%, P=0.03)$. Employing the SSS TOAST and imaging TOAST algorithms, there tended to be fewer cases in the undetermined categories for both races compared to the standard TOAST. 
TABLE 2: TOAST classifications of ischemic stroke subtypes by race.

\begin{tabular}{lccccccccc}
\hline & & TOAST & \multicolumn{3}{c}{ SSS-TOAST } & \multicolumn{3}{c}{ Imaging TOAST } \\
& Black & White & $P$ & Black & White & $P$ & Black & White & $P$ \\
\hline Cardioembolic, $n(\%)$ & $80(31)$ & $27(30)$ & 0.96 & $75(29)$ & $23(26)$ & 0.60 & $90(34)$ & $33(37)$ & 0.66 \\
Large artery, $n(\%)$ & $21(08)$ & $9(10)$ & 0.55 & $31(12)$ & $13(15)$ & 0.50 & $22(08)$ & $8(09)$ & 0.87 \\
Other, $n(\%)$ & $18(07)$ & $13(15)$ & 0.03 & $26(10)$ & $16(18)$ & 0.04 & $15(06)$ & $11(12)$ & 0.04 \\
Small vessel, $n(\%)$ & $28(11)$ & $9(10)$ & 0.87 & $33(13)$ & $12(13)$ & 0.84 & $30(11)$ & $9(10)$ & 0.72 \\
Undetermined, $n(\%)$ & $114(44)$ & $31(35)$ & 0.14 & $96(37)$ & $25(28)$ & 0.14 & $104(40)$ & $28(31)$ & 0.16 \\
\hline
\end{tabular}

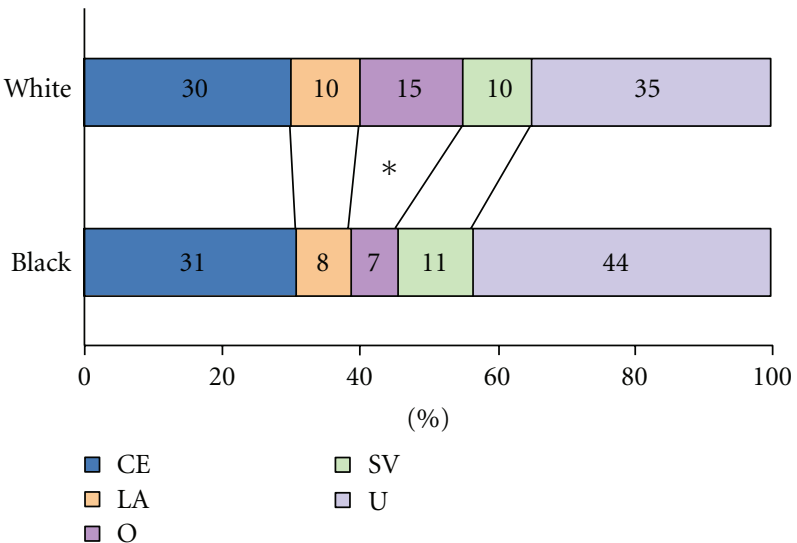

(a)

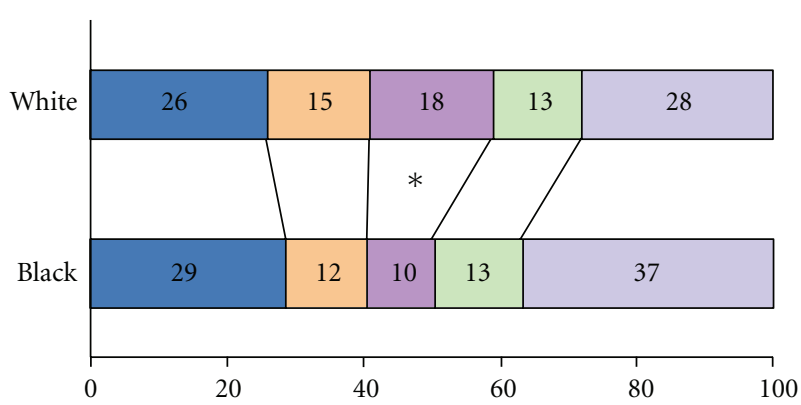

(\%)

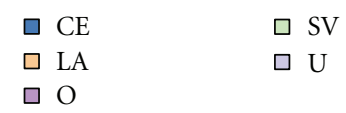

(b)

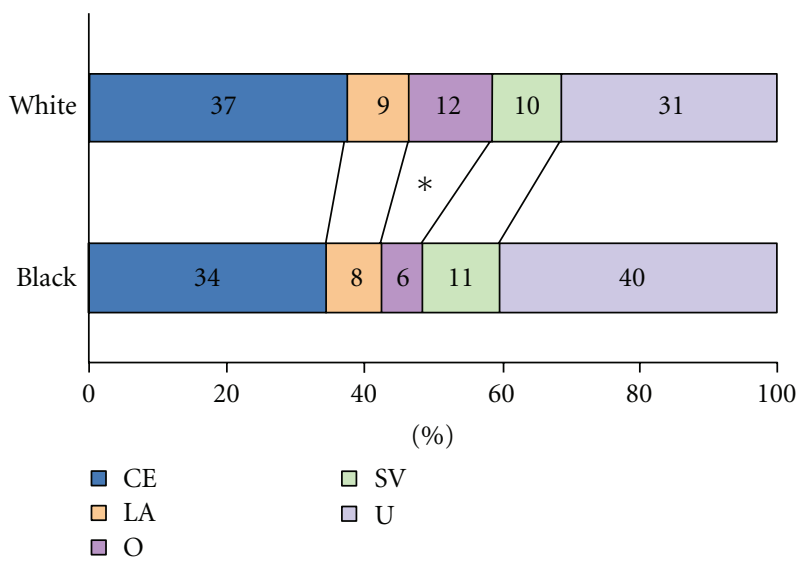

(c)

Figure 1: Proportions of stroke subtype by race for each classification system (*indicates statistical significance at a $P<0.05)$. (a) Standard TOAST. (b) SSS-TOAST. (c) Imaging TOAST.

Overall, in the white patient group, $30 \%$ were female; in the black patient group, $58 \%$ were female. There were no significant differences in etiology utilizing the standard TOAST etiology between white men, white women, black men, and black women. However, due to small numbers of "other" etiology in black men as compared to black women ( $1 \%$ versus $7 \%, P=0.06$ ), there was a trend towards significance.

For each classification system, the subcategories of stroke of undetermined etiology were classified differently and therefore could not be compared across systems. There were no significant differences by race in these subcategories for the standard TOAST or imaging TOAST, although there was a trend in both towards more black versus white patients with more than 1 etiology ( $14 \%$ versus $8 \%, P=0.12$ for standard TOAST, $13 \%$ versus $7 \%, P=0.09$ for imaging TOAST). For the SSS TOAST, there were no significant racial differences in the cryptogenic or other categories. There was, however, a trend towards more black stroke patients with incomplete workups ( $7 \%$ versus $2 \%, P=0.11$ ) and unclassified strokes (9\% versus $1 \%, P<0.01$ ) although the numbers are small. Of note, there was no significant difference in incomplete 
workup by race employing the standard TOAST system (11\% versus $8 \%, P=0.34$ ). There were no differences by race in completion of individual components of the overall workup.

\section{Discussion}

This analysis of the distribution of ischemic stroke subtype by race uniquely employed a diagnostic workup that included diffusion-weighted MR imaging in the vast majority of patients. Unlike prior studies with incomplete workups and/or CT-based imaging, the overwhelming majority of patients included in our analysis had a comprehensive hospital-based diagnostic workup, including echocardiography, telemetry monitoring, vessel imaging, and laboratory testing. We compared results using three approaches to ischemic stroke subtype classification: standard TOAST [11], SSS TOAST [12], and imaging-based TOAST [9]. Employing this comprehensive diagnostic approach, our study found a higher proportion of cardioembolic strokes and a smaller proportion of small vessel strokes in blacks (compared to prior studies) such that both blacks and whites had similar proportions of both subtypes. We also found that whites had a relatively small proportion of large artery strokes, a finding contrary to many previous studies. The main significant difference between groups was that the black cohort had significantly fewer strokes in the "strokes of other etiology" category. There are a number of possible explanations for the noted differences in the racial proportion of ischemic stroke subtypes. As noted above, the extent of the diagnostic workup may have differed. Alternatively, there may be geographic and community-based differences in risk factors and/or subtypes [13].

A number of prior studies have reported racial differences in stroke subtype proportions $[1,2,6,7,14]$. In the Northern Manhattan population, White et al. reported similar rates of small-vessel strokes in blacks and whites but a greater rate of the cardioembolic subtype in whites as compared to blacks [2]. Schneider reported similar findings in the Cincinnati population [1]. Markus et al. found a significantly greater proportion of lacunes in the British black population as compared to whites offset by a greater proportion of cardioembolic subtype in whites [8]. This study reports similar proportions of cardioembolic and lacunar strokes in both black and white cohorts employing diffusion-weighted MR imaging in the majority of patients.

An analysis of risk factors in our population showed that the black stroke cohort had significantly greater rates of diabetes and hypertension compared to whites, while the rates of coronary artery disease and hyperlipidemia were similar. In our cohort, the mean age did not differ by race; however there was a significant racial difference in gender between groups with $42 \%$ of the black cohort being male compared to $70 \%$ of the white cohort. Our population differs in risk factors and baseline characteristics from some prior reports in the fact that age was similar in both groups (whereas in most prior studies blacks have a younger age) and that there were more women in the black cohort.

Compared to the standard TOAST classification, the SSS TOAST classified more patients in the cardioembolic, large artery, small vessel, and other categories and fewer in the undetermined category for both groups, while the imaging TOAST classified more subjects only in the cardioembolic category. In our study, 79 patients (black and white) were found to have strokes of undetermined etiology under standard TOAST criteria. After utilizing, for instance, imaging TOAST criteria, the number of strokes of undetermined etiology decreased to 71 , a $10 \%$ change. The cardioembolic category had the greatest increase of cases, signaling a shift from an undetermined to a clearer etiology by using DWI MRI. This is likely due to the ability of DWI to detect multiple small acute lesions (not visualized on CT) in different vascular territories [15]. However, we point out that a substantial number of cases in each method of etiologic TOAST criteria still remained undetermined, despite the comprehensive diagnostic workup that patients received.

Accurate determination of stroke subtype on an individual level is important in order to appropriately tailor secondary prevention strategies. In particular, cardioembolic stroke is often most appropriately treated with anticoagulation rather than antiplatelet agents, particularly in the setting of atrial fibrillation. Recent studies have suggested that cardioembolism from atrial fibrillation may be underdiagnosed in general, but particularly so in the black population [16]. It is possible that a significant proportion of patients previously diagnosed with stroke of undetermined subtype may actually have had cardioembolism, and particularly in black stroke patients. Our study suggests that more research needs to be done to confirm and explain racial differences in stroke subtype, particularly in the category of "other etiology." One possibility is a racial or genetic susceptibility to certain types of stroke or hypercoagulable states.

Racial differences in the subcategory of "incomplete workup" using the SSS TOAST system is worth noting, although this difference did not reach statistical significance using the standard TOAST classification. A number of studies [17-20], but not all [21], have found important disparities in the extent of diagnostic evaluation in minority populations with stroke. A study of Michigan hospitals found that African American stroke patients were less likely to receive cardiac monitoring, dysphagia screening, smoking cessation counseling, or a CT within 25 minutes of hospital arrival. However, other aspects of the diagnostic evaluation including imaging of brain, vessels, and heart did not show a racial difference [17]. Tuhrim et al. reported that black stroke patients were less likely to receive complete diagnostic evaluations and less likely to receive appropriate secondary prevention measures [18]. These racial disparities in evaluation and treatment may be explained by differences in insurance or socioeconomic status, patient mistrust of the medical system, and/or clinician bias. Since all patients had standard order sets and the stroke team followed a prespecified pathway for diagnostic workup in this study, the underlying reason for disproportionally incomplete evaluations in the black population is unclear.

This study is limited by the fact that it was a hospitalbased retrospective chart review rather than a populationbased study. As with any hospital-based study, there is the potential for selection bias such that the population is not 
representative of the general population. However, Washington Hospital Center is the main community hospital for the region and receives over one-half of all strokes in the DC metropolitan area. Furthermore, a hospital-based study has the advantage of incorporating a more comprehensive and consistent diagnostic workup, which is crucial to an accurate determination of stroke subtype. Of note, we were not able to collect data on socioeconomic status or insurance. Future studies should include these variables in analyses, particularly studies evaluating the disparities in diagnostic assessments. Gender differences in our population may also have played a factor in the results we received. We had a much higher proportion of women in the black patient group $(58 \%)$ compared to the women in the white patient group $(30 \%)$. Given this was a retrospective study based upon a single hospital's experience, in future studies, a larger, more random sample would give more power to any differences found. We also used three different TOAST criteria to diagnose etiology of ischemic stroke, recognizing that other studies have used other forms of etiologic criteria, such as the modified NINDS scheme.

In conclusion, we found no difference in the frequency of ischemic stroke subtypes when employing a comprehensive diagnostic evaluation including diffusion-weighted MRI. This finding is particularly robust given the findings of high rates of hypertension and diabetes in our population-risk factors specifically associated with small-vessel strokes. In addition, our results suggest that the relatively high rate of cardioembolism may be underappreciated in black stroke patients and that diffusion-weighted MR imaging may be important in confirming this stroke subtype. Further research is required to better understand the racial differences in frequency of "stroke of other etiology" and to explore racial disparities in the extent of diagnostic evaluations.

\section{Acknowledgments}

This work was supported by NINDS/NCMHD (NINDS U54NS057405). This research was also supported in part by the Intramural Research Program of the NINDS, NIH. S. Song received support from the American Heart Association/Pharmaceutical Roundtable-Spina Outcomes Research Center and the University of California, Los Angeles, Resource Centers for Minority Aging Research Center for Health Improvement of Minority Elderly (RCMAR/CHIME) under NIH/NIA Grant P30-AG021684, and the content does not necessarily represent the official views of the NIA or the NIH. The authors wish to acknowledge the NIH Stroke Natural History Investigators and wish to thank Dr. Dorothy Edwards for her critical review of the paper.

\section{References}

[1] A. T. Schneider, B. Kissela, D. Woo et al., "Ischemic stroke subtypes: a population-based study of incidence rates among blacks and whites," Stroke, vol. 35, no. 7, pp. 1552-1556, 2004.

[2] H. White, B. Boden-Albala, C. Wang et al., "Ischemic stroke subtype incidence among whites, blacks, and Hispanics: the northern Manhattan study," Circulation, vol. 111, no. 10, pp. 1327-1331, 2005.

[3] R. L. Sacco, B. Boden-Albala, R. Gan et al., "Stroke incidence among white, black, and Hispanic residents of an urban community: the Northern Manhattan Stroke Study," American Journal of Epidemiology, vol. 147, no. 3, pp. 259-268, 1998.

[4] T. Ohira, E. Shahar, L. E. Chambless, W. D. Rosamond, T. H. Mosley, and A. R. Folsom, "Risk factors for ischemic stroke subtypes: the atherosclerosis risk in communities study," Stroke, vol. 37, no. 10, pp. 2493-2498, 2006.

[5] D. Woo, J. Gebel, R. Miller et al., "Incidence rates of first-ever ischemic stroke subtypes among blacks: a population-based study," Stroke, vol. 30, no. 12, pp. 2517-2522, 1999.

[6] G. Friday, S. M. Lai, M. Alter et al., "Stroke in the Lehigh valley: racial/ethnic differences," Neurology, vol. 39, no. 9, pp. 11651168, 1989.

[7] R. L. Sacco, D. E. Kargman, Q. Gu, and M. C. Zamanillo, "Race-ethnicity and determinants of intracranial atherosclerotic cerebral infarction: the Northern Manhattan stroke study," Stroke, vol. 26, no. 1, pp. 14-20, 1995.

[8] H. S. Markus, U. Khan, J. Birns et al., "Differences in stroke subtypes between black and white patients with stroke: the South London Ethnicity and Stroke Study," Circulation, vol. 116, no. 19, pp. 2157-2164, 2007.

[9] L. J. Lee, C. S. Kidwell, J. Alger, S. Starkman, and J. L. Saver, "Impact on stroke subtype diagnosis of early diffusion-weighted magnetic resonance imaging and magnetic resonance angiography," Stroke, vol. 31, no. 5, pp. 1081-1089, 2000.

[10] T. Wessels, C. Röttger, M. Jauss, M. Kaps, H. Traupe, and E. Stol, "Identification of embolic stroke patterns by diffusionweighted MRI in clinically defined lacunar stroke syndromes," Stroke, vol. 36, no. 4, pp. 757-761, 2005.

[11] H. P. Adams Jr., B. H. Bendixen, L. J. Kappelle et al., "Classification of subtype of acute ischemic stroke: definitions for use in a multicenter clinical trial," Stroke, vol. 24, no. 1, pp. 35-41, 1993.

[12] H. Ay, K. L. Furie, A. Singhal, W. S. Smith, A. G. Sorensen, and W. J. Koroshetz, "An evidence-based causative classification system for acute ischemic stroke," Annals of Neurology, vol. 58, no. 5, pp. 688-697, 2005.

[13] C. Hajat, R. Dundas, J. A. Stewart et al., "Cerebrovascular risk factors and stroke subtypes: differences between ethnic groups," Stroke, vol. 32, no. 1, pp. 37-42, 2001.

[14] R. J. Wityk, D. Lehman, M. Klag, J. Coresh, H. Ahn, and B. Litt, "Race and sex differences in the distribution of cerebral atherosclerosis," Stroke, vol. 27, no. 11, pp. 1974-1980, 1996.

[15] R. E. Burgess and C. S. Kidwell, "Use of MRI in the assessment of patients with stroke," Current Neurology and Neuroscience Reports, vol. 11, no. 1, pp. 28-34, 2011.

[16] J. F. Meschia, P. Merrill, E. Z. Soliman et al., "Racial disparities in awareness and treatment of atrial fibrillation: the REasons for geographic and racial differences in stroke (REGARDS) study," Stroke, vol. 41, no. 4, pp. 581-587, 2010.

[17] B. S. Jacobs, G. Birbeck, A. J. Mullard et al., "Quality of hospital care in African American and white patients with ischemic stroke and TIA," Neurology, vol. 66, no. 6, pp. 809-814, 2006.

[18] S. Tuhrim, A. Cooperman, M. Rojas et al., "The association of race and sex with the underuse of stroke prevention measures," Journal of Stroke and Cerebrovascular Diseases, vol. 17, no. 4, pp. 226-234, 2008.

[19] J. B. Mitchell, D. J. Ballard, D. B. Matchar, J. P. Whisnant, and G. P. Samsa, "Racial variation in treatment for transient ischemic attacks: impact of participation by neurologists," Health Services Research, vol. 34, no. 7, pp. 1413-1428, 2000. 
[20] E. Z. Oddone, R. D. Horner, R. Sloane et al., "Race, presenting signs and symptoms, use of carotid artery imaging, and appropriateness of carotid endarterectomy," Stroke, vol. 30, no. 7, pp. 1350-1356, 1999.

[21] L. B. Goldstein, D. B. Matchar, J. Hoff-Lindquist, G. P. Samsa, and R. D. Horner, "Veterans Administration Acute Stroke (VAST) study: lack of race/ethnic-based differences in utilization of stroke-related procedures or services," Stroke, vol. 34, no. 4, pp. 999-1003, 2003. 


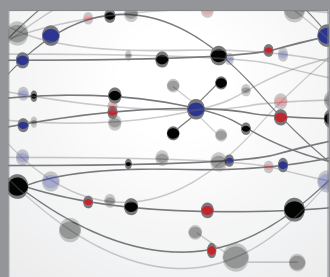

The Scientific World Journal
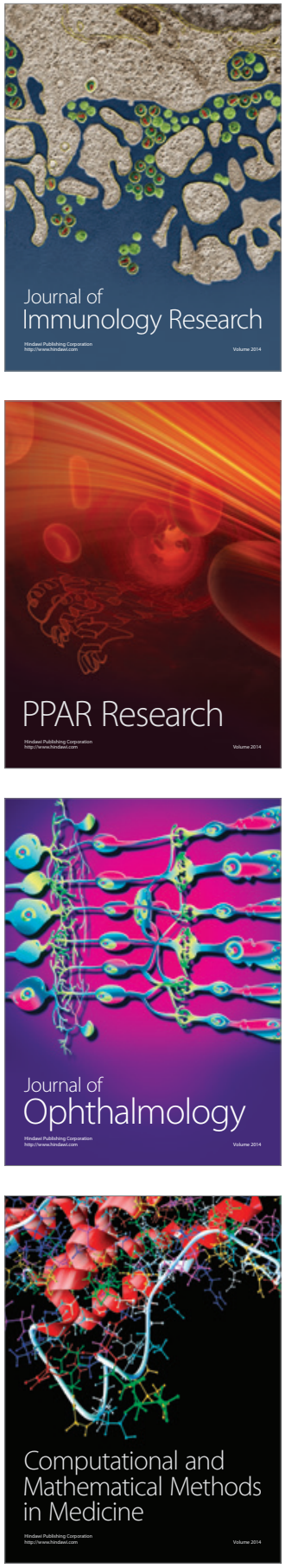

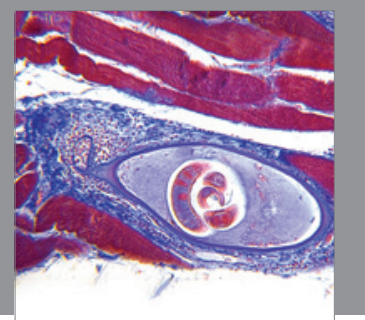

Gastroenterology

Research and Practice
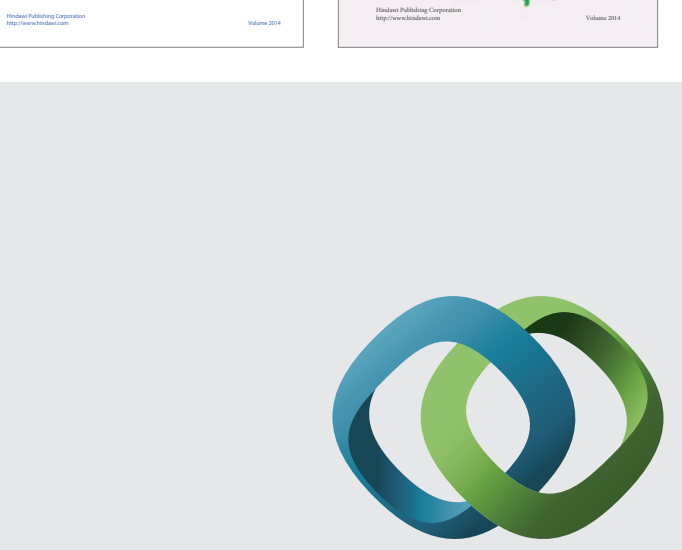

\section{Hindawi}

Submit your manuscripts at

http://www.hindawi.com
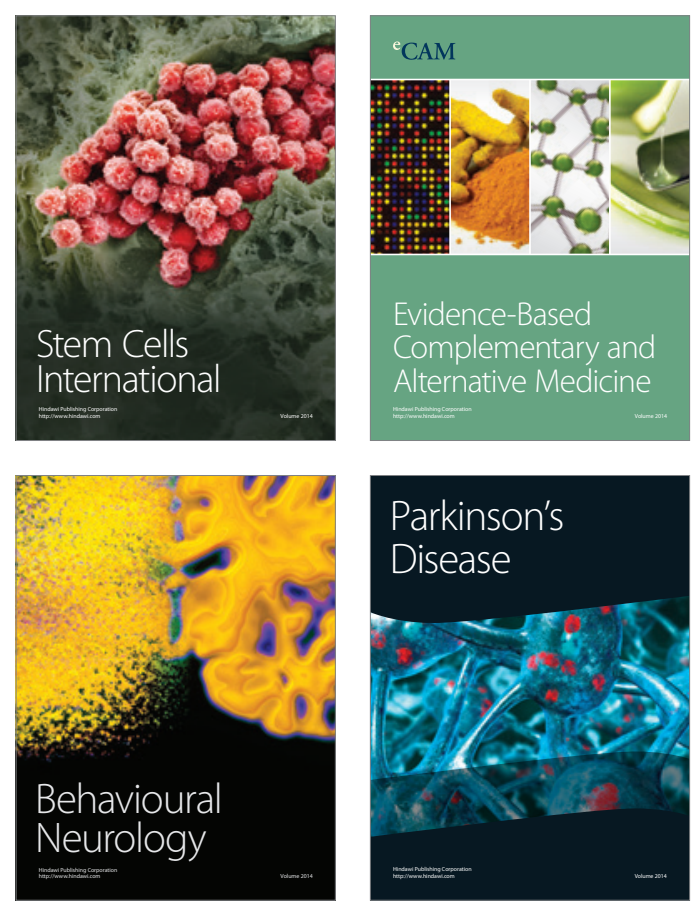

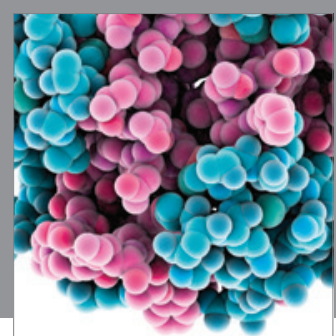

Journal of
Diabetes Research

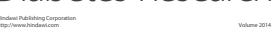

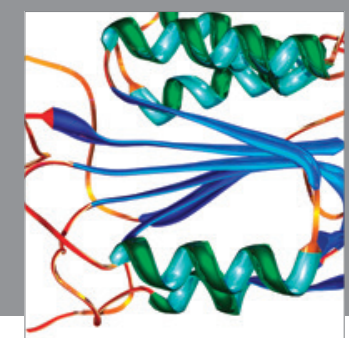

Disease Markers
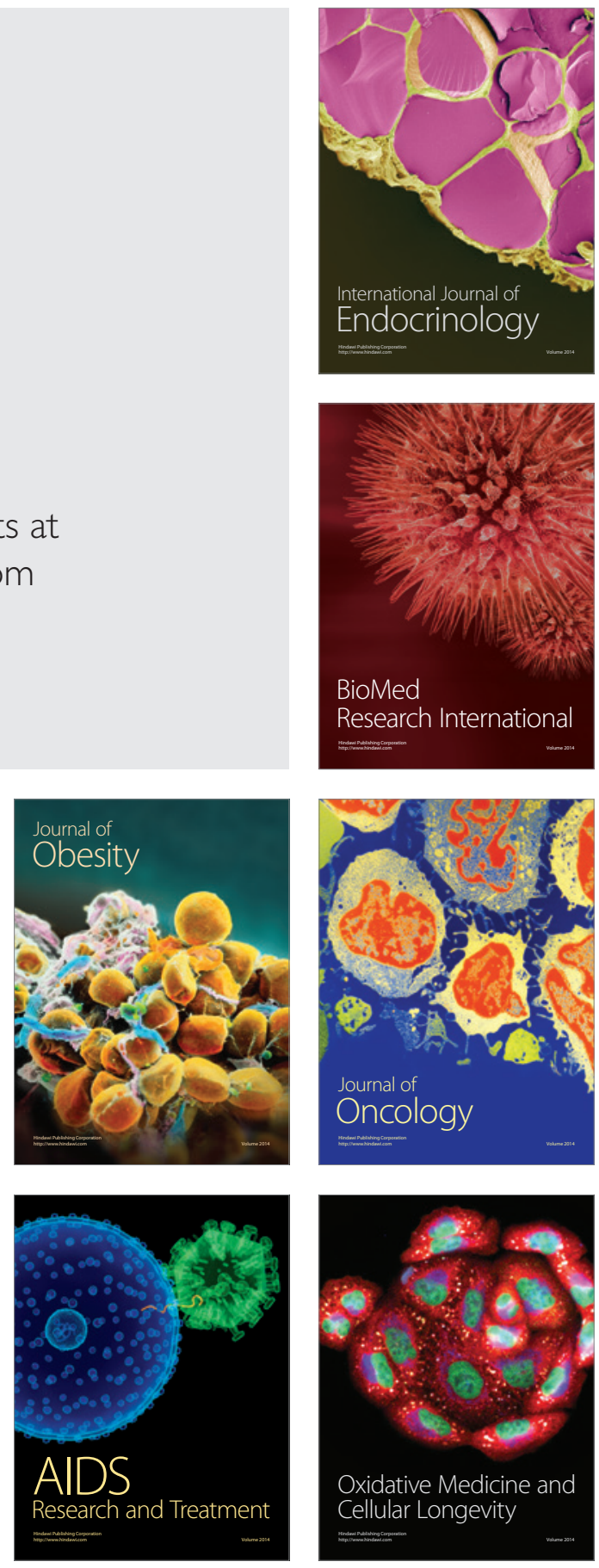